\title{
Noise-Intensity Fluctuation in Langevin Model and Its Higher-Order Fokker-Planck Equation
}

\author{
Yoshihiko Hasegawa* \\ Department of Biophysics and Biochemistry, Graduate School of Science, \\ The University of Tokyo, Tokyo 113-0033, Japan \\ Masanori Arita \\ Department of Biophysics and Biochemistry, Graduate School of Science, \\ The University of Tokyo, Tokyo 113-0033, Japan and \\ Institute for Advanced Biosciences, Keio University, Yamagata 997-0035, Japan
}

(Dated: November 26, 2010)

\begin{abstract}
In this paper, we investigate a Langevin model subjected to stochastic intensity noise (SIN), which incorporates temporal fluctuations in noise-intensity. We derive a higher-order FokkerPlanck equation (HFPE) of the system, taking into account the effect of SIN by the adiabatic elimination technique. Stationary distributions of the HFPE are calculated by using the perturbation expansion. We investigate the effect of SIN in three cases: (a) parabolic and quartic bistable potentials with additive noise, (b) a quartic potential with multiplicative noise, and (c) a stochastic gene expression model. We find that the existence of noise intensity fluctuations induces an intriguing phenomenon of a bimodal-to-trimodal transition in probability distributions. These results are validated with Monte Carlo simulations.
\end{abstract}

PACS numbers: 05.10.Gg, 05.40.Ca

Keywords: Stochastic process, Superstatistics, Stochastic volatility, Adiabatic elimination, Higher-order Fokker-Planck equation

*Electronic address: hasegawa@cb.k.u-tokyo.ac.jp 


\section{INTRODUCTION}

Many real-world systems are inhomogeneous and fluctuant. Stochastic processes are often used for modeling such fluctuant systems in many fields, including physics, biology, and chemistry. The dynamics in these systems can be described by a Langevin equation given by

$$
\frac{d x}{d t}=f(x)+g(x) \xi_{x}(t)
$$

where $f(x)=-\partial_{x} U(x), U(x)$ denotes a potential, $g(x)$ is an arbitrary function of $x$, and $\xi_{x}(t)$ is the white Gaussian noise with correlation $\left\langle\xi_{x}(t) \xi_{x}\left(t^{\prime}\right)\right\rangle=2 D_{x} \delta\left(t-t^{\prime}\right)$. Although white noise can reflect microscale properties of fluctuations, it is uniform when seen from mesoscopic or macroscopic time scales [Fig. 1(a)]. One widely used approach for incorporating mesoscopic or macroscopic inhomogeneity in noise is colored noise, where the white noise $\xi_{x}(t)$ in Eq. (1) is replaced by $z(t)$ with the Ornstein-Uhlenbeck process:

$$
\frac{d z}{d t}=-\frac{z}{\tau}+\frac{\xi_{z}(t)}{\tau}
$$

where $\xi_{z}(t)$ expresses the white Gaussian noise $\left[\left\langle\xi_{z}(t) \xi_{z}\left(t^{\prime}\right)\right\rangle=2 D_{z} \delta\left(t-t^{\prime}\right)\right]$. Equation (2) yields colored noise with the finite correlation time $\tau$, that is, $\left\langle z(t) z\left(t^{\prime}\right)\right\rangle=$ $\left(D_{z} / \tau\right) \exp \left\{-\left|t-t^{\prime}\right| / \tau\right\}$. The existence of correlation time in the noise sources can induce many phenomena, such as resonant activation [1] and noise-enhanced stability [2 6].

In the present paper, we deal with mesoscopic time-scale inhomogeneity in a way other than with colored noise; here, we consider temporal noise-intensity fluctuations. We assume that the noise intensity in Eq. (11) is not constant but modulated by the Ornstein-Uhlenbeck process. Our model is described by the following coupled Langevin equations instead of Eq. (11):

$$
\begin{aligned}
& \frac{d x}{d t}=f(x)+g(x) s \xi_{x}(t) \\
& \frac{d s}{d t}=-\gamma(s-\alpha)+\sqrt{\gamma} \xi_{s}(t) .
\end{aligned}
$$

Here, $\xi_{s}(t)$ is the white Gaussian noise $\left[\left\langle\xi_{s}(t) \xi_{s}\left(t^{\prime}\right)\right\rangle=2 D_{s} \delta\left(t-t^{\prime}\right)\right]$, and $\gamma$ and $\alpha$ are the relaxation rate and the mean of the Ornstein-Uhlenbeck process, respectively. The intensitymodulated noise term $s \xi_{x}(t)$ in Eq. (3) is herein called stochastic intensity noise (SIN) [Fig. 1(b)]. This point of view was originally introduced in Heston's stochastic volatility models in financial engineering [7] and has since been analyzed in econophysics [8]. With $f(x) \propto x$ 
and $g(x) \propto x$, Eqs. (3) and (44) are similar to those in the Heston model, where the variance of noise is governed by the Feller process (also referred to as the square-root process or the Cox-Ingersoll-Ross process). Escape events in the Heston model were studied in Ref. [9] using a cubic potential. Note that the variable $s$ in Eqs. (3) and (4) takes negative as well as positive values since our model can be considered as white noise with multiplicative term $s$, which is in contrast with the positive variance in the Heston model [7]. In physics, superstatistics includes the concept of temporal and/or spatial environmental fluctuations [10 14]. Superstatistics was originally introduced, under specific conditions, to account for asymptotic power-law distributions (e.g., q-exponential distributions and q-Gaussian distributions) that emerge as maximizers of non-additive (Tsallis) entropy [15, 16]. Superstatistics has since been applied to the interpretation of quasi-equilibrium thermodynamics, and concepts of superstatistics have also been applied to stochastic processes [11, 13, 17-[20]. In particular, Ref. [17] extended superstatistics to the path-integral representation and showed that some stochastic models can be covered by this representation. A direct connection between Tsallis statistics and financial stochastic processes was indicated in Ref. [21].

In many biological and chemical processes, the relaxation time of $x$ may be larger than that of environmental fluctuations (noise-intensity processes). This is the case in stochastic gene expression models in which the decay time of $x$ is on the order of minutes [22] (Sec. 5.3). Bearing this fact in mind, we will investigate systems driven by SIN with faster decay time compared with that of $x(\gamma \gg 1)$. Furthermore, in real-world systems, we expect that the $f(x)$ in Eq. (3) is often given by a complex nonlinear function and is also accompanied by nontrivial multiplicative noise expressed by an appropriate $g(x)$. These properties are different from those for financial engineering. In order to obtain the probability distribution $P(x, t)$, we use adiabatic elimination with eigenfunction expansion [23]. We obtain a higherorder Fokker-Planck equation (HFPE) with higher-order derivatives, not included in the conventional Fokker-Planck equation (FPE). We calculate stationary distributions of the systems described by the HFPE by using the perturbation expansion.

To investigate the effects of SIN, we consider stationary distributions for three cases: (a) parabolic and quartic bistable potentials with additive noise, (b) a quartic potential with multiplicative noise and (c) a stochastic gene expression model. In the additive noise case (a), we show that SIN changes the stationary distribution from exponential forms (the Boltzmann-Gibbs distribution) to non-exponential forms. At the same time, the stationary 

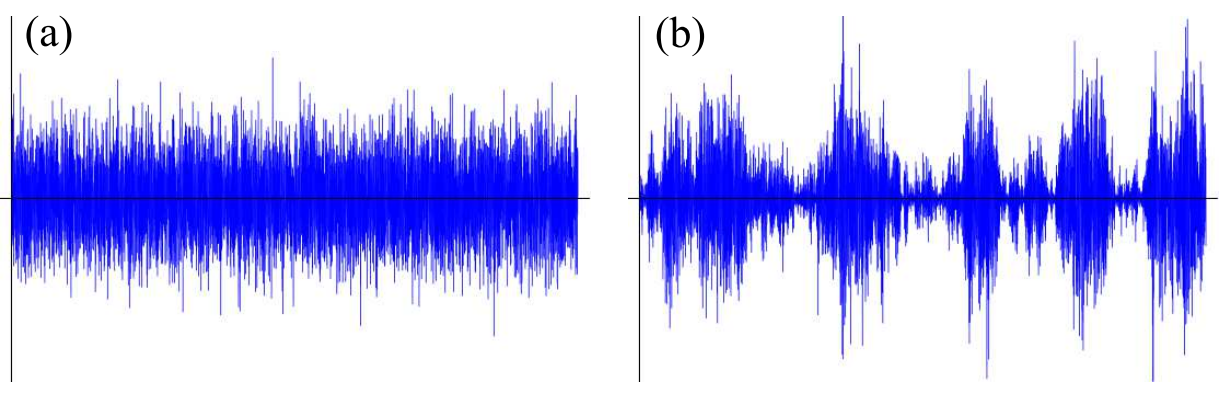

FIG. 1: (Color online) Intuitive paths of (a) white noise and (b) SIN whose intensity is governed by the Ornstein-Uhlenbeck process [Eq. (41)].

distributions of case (a) are sharpened because of noise-intensity fluctuations. In case (b), we show that the existence of noise-intensity fluctuations induces the transition of distributions: the stationary distributions are uni-, bi-, or trimodal, depending on the SIN parameters. It is important to note that the trimodal distribution does not emerge under white noise. In case (c) which we call the gene expression model, a nonlinear function is given as a drift term (change in expression levels). Thus, case (c) shows that our approximation scheme can be applied to general configurations that include non-trivial drift terms.

The remainder of this paper is organized as follows: In Sec. 2, we describe the model proposed in this paper. Adiabatic elimination with eigenfunction expansion is applied to the model in Sec. 3 (details of the derivation of the HFPE are explained in the Appendix). In Sec. 4, we proceed to the calculation of the stationary distributions of the obtained HFPE by using of the perturbation expansion. In Sec. 5, we investigate effects of SIN in the three cases (a), (b), and (c) mentioned above. In Sec. 6, we analyze effects of higher-order derivatives in the HFPE on positivity and moments of distribution functions, and also consider the opposite case, in which a decay time of the noise-intensity fluctuations is very slow $(\gamma \rightarrow 0)$. Finally, we give the conclusions in Sec. 7.

\section{THE MODEL}

We consider the Langevin equations given by Eqs. (3) and (44). Since Eq. (4) does not depend on $x$, the stationary distribution $P_{s t}(s)$ of $s$ is easily obtained:

$$
P_{s t}(s)=\frac{1}{\sqrt{2 \pi D_{s}}} \exp \left\{-\frac{(s-\alpha)^{2}}{2 D_{s}}\right\} .
$$


Time evolution of the probability distribution $P(x, s ; t)$ is given by

$$
\frac{\partial}{\partial t} P(x, s ; t)=\mathrm{L}_{\mathrm{FP}}(x, s) P(x, s ; t)
$$

where $\mathrm{L}_{\mathrm{FP}}(x, s)$ is a FPE operator composed of

$$
\mathrm{L}_{\mathrm{FP}}(x, s)=\mathrm{L}_{x}(x, s)+\gamma \mathrm{L}_{s}(s)
$$

with

$$
\begin{gathered}
\mathrm{L}_{x}(x, s)=-\frac{\partial}{\partial x}\left\{f(x)+D_{x} g(x) g^{\prime}(x) s^{2}\right\}+D_{x} s^{2} \frac{\partial^{2}}{\partial x^{2}} g(x)^{2}, \\
\mathrm{~L}_{s}(s)=\frac{\partial}{\partial s}(s-\alpha)+D_{s} \frac{\partial^{2}}{\partial s^{2}} .
\end{gathered}
$$

We employ Stratonovich's calculus because it is expected to be more relevant to physical applications than Itô's [24]. We calculate a projected time evolution equation of $P(x ; t)=$ $\int d s P(x, s ; t)$ that can be applied to general configurations.

\section{ELIMINATION OF THE FAST VARIABLE}

In this section, we eliminate $s$ from Eq. (6) by using adiabatic elimination under the assumption that $\gamma \gg 1$ (i.e., the decay time of $s$ is much faster than that of $x$ ). Adiabatic elimination has its origin in the Born-Oppenheimer approximation. Because of its usefulness, adiabatic elimination has been applied to many stochastic systems [25, 26]. In stochastic volatility models, $\gamma \rightarrow 0$ adiabatic elimination has been applied [27, 28]. These models take advantage of the fact that noise intensity (volatility) changes on a macroscopic time scale in financial markets (this description agrees with Beck's superstatistical Brownian motion [13]). In these studies, the obtained solutions do not explicitly include $\gamma$ as a parameter. In the present paper, we consider the $\gamma \gg 1$ case and derive a HFPE containing $O\left(\gamma^{-1}\right)$ terms by using adiabatic elimination with eigenfunction expansion [23].

By applying adiabatic elimination up to $O\left(\gamma^{-1}\right)$, we obtain the following HFPE (see the Appendix for the derivation):

$$
\frac{\partial}{\partial t} P(x ; t)=\left\{-\frac{\partial}{\partial x} f(x)+Q \triangle_{g}+\frac{R}{\gamma} \triangle_{g}^{2}\right\} P(x ; t),
$$

with

$$
\triangle_{g}=\frac{\partial^{2}}{\partial x^{2}} g(x)^{2}-\frac{\partial}{\partial x} g^{\prime}(x) g(x)
$$


where $Q$ and $R$ in Eq. (10) are defined as follows:

$$
\begin{gathered}
Q=D_{x}\left(D_{s}+\alpha^{2}\right), \\
R=D_{x}^{2} D_{s}\left(4 \alpha^{2}+D_{s}\right) .
\end{gathered}
$$

For the case of additive noise [i.e., $g(x)=1$ ], Eq. (10) reduces to

$$
\frac{\partial}{\partial t} P(x ; t)=\left\{-\frac{\partial}{\partial x} f(x)+Q \frac{\partial^{2}}{\partial x^{2}}+\frac{R}{\gamma} \frac{\partial^{4}}{\partial x^{4}}\right\} P(x ; t) .
$$

Because Eqs. (10) and (12) have derivatives of orders higher than two, Eqs. (10) and (12) are referred to as the HFPE. In systems driven by colored noise, adiabatic elimination up to $O(\tau)$ can be expressed by the conventional FPE [23] [see Eq. (2)]. On the other hand, $O\left(\gamma^{-1}\right)$ terms are accompanied by non-FPE terms in our SIN case. Therefore, in order to incorporate the effect of $\gamma$, we must use the HFPE form of Eq. (10). For $\gamma^{-1} \rightarrow 0$, the last term of Eq. (10) vanishes, and we obtain FPE:

$$
\frac{\partial}{\partial t} P(x ; t)=-\frac{\partial}{\partial x}\left\{f(x)+Q g^{\prime}(x) g(x)\right\} P(x ; t)+Q \frac{\partial^{2}}{\partial x^{2}} g(x)^{2} P(x ; t) .
$$

Eq. (13) corresponds to conventional $(\gamma \rightarrow \infty)$ adiabatic elimination. $Q$ plays the role of the effective noise intensity of the corresponding white Gaussian noise process.

\section{STATIONARY DISTRIBUTION}

In many practical cases, stationary distributions play important roles. In this section, we

calculate the stationary distribution $P_{s t}(x)$ of Eq. (10), which yields the following differential equation:

$$
-\frac{f}{Q} P_{s t}(x)+\left(\frac{\partial}{\partial x} g^{2}-g^{\prime} g\right) P_{s t}(x)+\varepsilon\left(\frac{\partial}{\partial x} g^{2}-g^{\prime} g\right) \frac{\partial}{\partial x}\left(\frac{\partial}{\partial x} g^{2}-g^{\prime} g\right) P_{s t}(x)=0,
$$

where

$$
\varepsilon=\frac{R}{\gamma Q}=\frac{D_{x} D_{s}\left(4 \alpha^{2}+D_{s}\right)}{\gamma\left(D_{s}+\alpha^{2}\right)}
$$

It is easy to see that a solution of Eq. (14) for $\varepsilon=0$ is the stationary distribution of Eq. (13). Thus, for $\varepsilon \ll 1$, it is expected that a solution of Eq. (14) can be approximated by the perturbation from the stationary distribution for $\varepsilon=0$. In solving Eq. (14), we adopt the perturbation expansion [29-32], given by

$$
P_{s t}(x)=\Pi_{0}(x)+\varepsilon \Pi_{1}(x)+\varepsilon^{2} \Pi_{2}(x)+\cdots
$$


We specifically define the following truncated first-order approximation:

$$
P_{s t}^{(1)}(x)=\Pi_{0}(x)+\varepsilon \Pi_{1}(x),
$$

where $\Pi_{0}(x)$ is the stationary distribution of the unperturbed case [Eq. (13)], and $\Pi_{1}(x)$ corresponds to the first-order correction term. Substituting Eq. (16) into Eq. (14) and comparing the order of $\varepsilon$ up to $O(\varepsilon)$, we obtain

$$
\begin{aligned}
& O(1) \quad\left\{\frac{f(x)}{Q}+g^{\prime}(x) g(x)\right\} \Pi_{0}(x)=\frac{\partial}{\partial x} g(x)^{2} \Pi_{0}(x), \\
& O(\varepsilon) \quad\left\{\frac{f(x)}{Q}+g^{\prime}(x) g(x)\right\} \Pi_{1}(x)=\frac{\partial}{\partial x} g(x)^{2} \Pi_{1}(x)+\phi(x),
\end{aligned}
$$

where

$$
\phi(x)=\left\{\frac{\partial}{\partial x} g(x)^{2}-g^{\prime}(x) g(x)\right\} \frac{\partial}{\partial x}\left\{\frac{\partial}{\partial x} g(x)^{2}-g^{\prime}(x) g(x)\right\} \Pi_{0}(x) .
$$

From Eq. (17), $\Pi_{0}(x)$ is given by

$$
\Pi_{0}(x)=\frac{1}{Z|g(x)|} \exp \left(\frac{1}{Q} \int^{x} d v \frac{f(v)}{g(v)^{2}}\right),
$$

where $Z$ is a normalizing term $\left[\int d x \Pi_{0}(x)=1\right]$. Since Eq. (18) is a first-order differential equation, $\Pi_{1}(x)$ can be obtained analytically in many cases, as will be discussed in Sec. 5 .

The perturbation expansion yields reliable results for $\varepsilon \ll 1$. Combining the approximation condition of adiabatic elimination and the perturbation expansion, it can be concluded that we are able to calculate the stationary distribution by Eq. (16) for systems with $\gamma \gg 1$ and $\varepsilon \ll 1$.

\section{MODEL CALCULATIONS}

We apply the approximation method obtained in Sec. 4 to three cases: parabolic and quartic bistable potentials with additive noise (Sec. 5.1), a quartic potential with multiplicative noise (Sec. 5.2), and a stochastic gene expression model (Sec. 5.3). We analyze

effects of noise-intensity fluctuations on shapes of the stationary distributions. We also carried out Monte Carlo (MC) simulations to determine the reliability of our approximation. In the following, we show results of three calculation methods:

\section{- HFPE stationary distribution}

The stationary distribution of the HFPE [Eq. (10)] is shown using the perturbation 
expansion explained in previous sections including the correction term $\Pi_{1}(x)$. This is an $O\left(\gamma^{-1}\right)$ approximation.

\section{- FPE stationary distribution}

The stationary distribution of the FPE [Eq. (13)] is shown. This is identical to $\Pi_{0}(x)$ and does not include the effect of noise-intensity fluctuations. This case corresponds to $\gamma^{-1} \rightarrow 0$ adiabatic elimination.

\section{- MC simulation}

We have employed a simple Euler-forward scheme with a time step size $\Delta t=10^{-6}$ (for details of the algorithm, readers may refer to Sec. 3.6 of Ref. [33]). We have used $N=5 \times 10^{7}$ points to calculate empirical distributions. For all the line-symmetric potentials, MC data are symmetrized with respect to $x=0$.

For evaluating quantitatively the reliability of HFPE stationary distributions, we calculate the root-mean-square (RMS) distance between the HFPE (or FPE) and MC distributions. We first divide an interval of $x$ (window of each figure) into $M$ points $(M=100$ ), each of which is denoted by $x_{i}$. Let $\widehat{P}_{i}$ and $P_{s t}\left(x_{i}\right)$ be density values of the MC and HFPE (FPE) distributions at $x_{i}$, respectively. RMS is defined by

$$
\mathrm{RMS}=\sqrt{\frac{1}{M} \sum_{i=1}^{M}\left(\widehat{P}_{i}-P_{s t}\left(x_{i}\right)\right)^{2}} .
$$

\subsection{Parabolic and Quartic Potentials with Additive Noise}

We investigate effects of additive SIN in parabolic and quartic bistable potentials. We first calculate the stationary distribution of the parabolic potential $\left[U(x)=x^{2} / 2\right]$ with additive SIN $[g(x)=1]$. According to Eqs. (17) and (18) $), \Pi_{0}(x)$ and $\Pi_{1}(x)$ are given as follows:

$$
\begin{gathered}
\Pi_{0}(x)=\sqrt{\frac{1}{2 \pi Q}} \exp \left(-\frac{x^{2}}{2 Q}\right), \\
\Pi_{1}(x)=\sqrt{\frac{1}{2 \pi Q}}\left(\frac{x^{4}}{4 Q^{3}}-\frac{3 x^{2}}{2 Q^{2}}\right) \exp \left(-\frac{x^{2}}{2 Q}\right)+C \exp \left(-\frac{x^{2}}{2 Q}\right),
\end{gathered}
$$

where $C$ is determined by the normalization condition $\left[\int d x P_{s t}^{(1)}(x)=1\right]$ :

$$
C=\frac{3}{4 \sqrt{2 \pi} Q^{3 / 2}}
$$


Substituting Eqs. (19) and (20) in Eq. (16), we obtain the stationary distribution $P_{s t}^{(1)}(x)$.

Figure 2 shows stationary distributions for the parabolic potential case calculated by HFPE (solid lines), FPE (dashed lines), and MC simulation (circles). We also show the RMS distance between the HFPE (FPE) and MC distributions in Fig. 2(a), (b), and (c). We see that the densities of the HFPE at $x=0$ are higher than those of FPE under the existence of noise-intensity fluctuations. Because the stationary distributions with noise-intensity fluctuations are approximately realized as superposition of many Gaussian distributions with different variances, those with smaller variance make the stationary distributions sharper. It is important to note that the stationary distributions of the HFPE (solid lines) are not Gaussian. This non-Gaussianity is derived from the existence of higher-order derivatives than the second in the HFPE (see Sec. 6.1). From the RMS values, we see that the distance between the HFPE and MC distributions is smaller than that between FPE and MC, indicating a better agreement of HFPE distributions. This result supports the reliability of our approximation scheme. The HFPE stationary distribution of $\gamma=15$ [Fig. 2(a)] exhibits better agreement than the $\gamma=5$ case [Fig. 2(c)] because larger $\gamma$ yields better approximation for the adiabatic elimination technique. The inset in Fig. 2(a) shows a log-scale plot for the $x \geq 0$ region, showing that the HFPE stationary distribution has fatter tails, which in turn implies that the existence of noise-intensity fluctuations makes distributions fatter (see Sec. 6.1). Furthermore, the HFPE stationary distribution exhibits better agreement with the MC simulations than the FPE in tail areas, indicating that our approximation scheme offers reliable results even in tail areas for sufficiently large $\gamma$ and sufficiently small $\varepsilon$.

We next calculate the stationary distributions of the quartic bistable potential $[U(x)=$ $\left.x^{4} / 4-x^{2} / 2\right]$ driven by additive $\operatorname{SIN}[g(x)=1]$. Because bistable potentials can represent switching dynamics, they are very important in many fields. $\Pi_{0}(x)$ and $\Pi_{1}(x)$ are given by

$$
\begin{gathered}
\Pi_{0}(x)=\frac{1}{Z} \exp \left\{-\frac{1}{Q}\left(\frac{x^{4}}{4}-\frac{x^{2}}{2}\right)\right\}, \\
\Pi_{1}(x)=\frac{x^{2}}{Z Q^{3}}\left\{3 Q^{2}-\frac{3}{2}\left(x^{2}-1\right)^{2} Q+x^{2}\left(\frac{x^{6}}{10}-\frac{3 x^{4}}{8}+\frac{x^{2}}{2}-\frac{1}{4}\right)\right\} \\
\times \exp \left\{-\frac{1}{Q}\left(\frac{x^{4}}{4}-\frac{x^{2}}{2}\right)\right\}+C \exp \left\{-\frac{1}{Q}\left(\frac{x^{4}}{4}-\frac{x^{2}}{2}\right)\right\},
\end{gathered}
$$

with

$$
Z=\frac{\pi}{2} \exp \left(\frac{1}{8 Q}\right)\left\{I_{-\frac{1}{4}}\left(\frac{1}{8 Q}\right)+I_{\frac{1}{4}}\left(\frac{1}{8 Q}\right)\right\}
$$



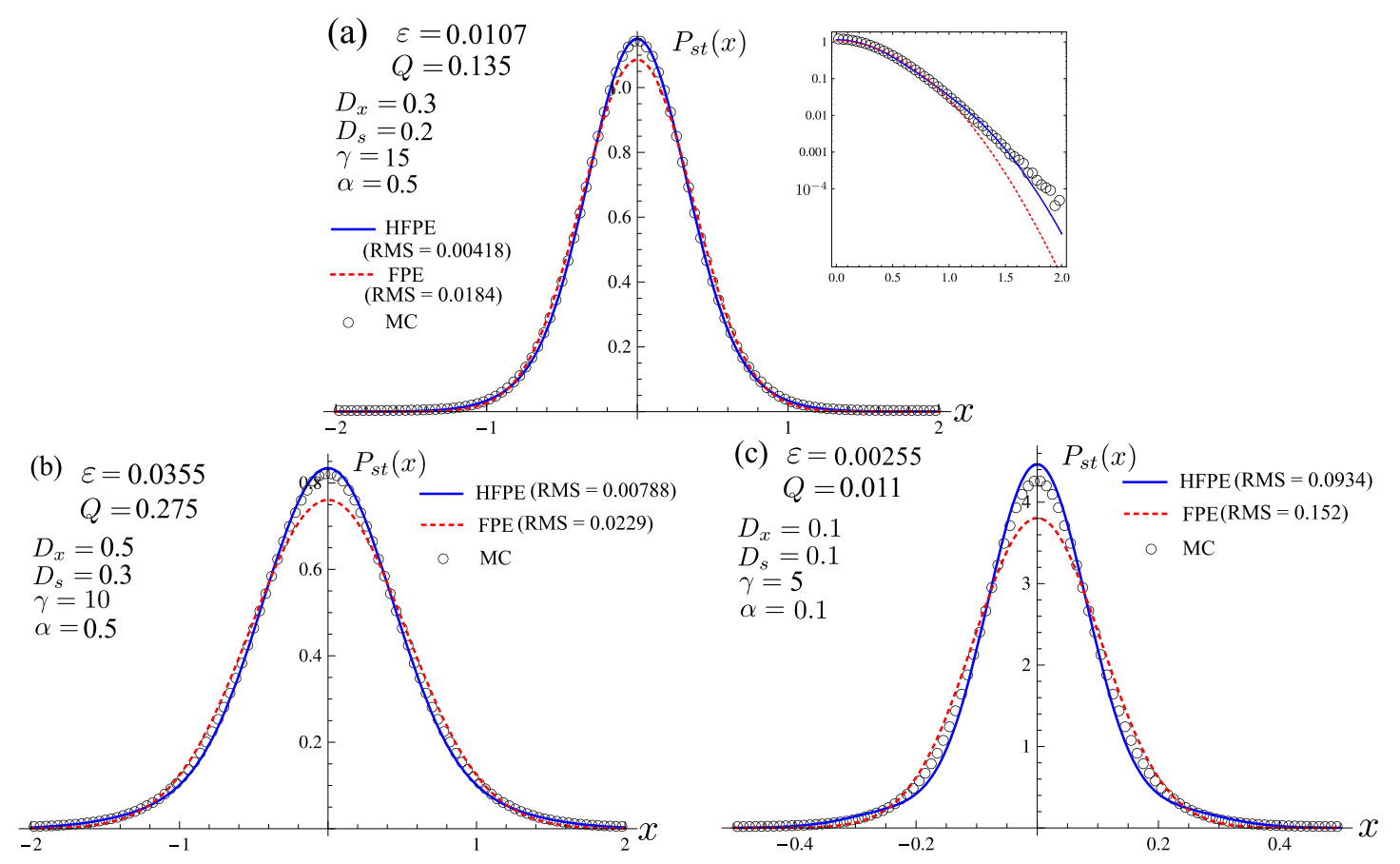

FIG. 2: (Color online) Stationary distributions for the parabolic potential with additive noise calculated by HFPE (solid lines), FPE (dashed lines), and MC (circles). Parameter values are (a) $D_{x}=0.3, D_{s}=0.2, \gamma=15$, and $\alpha=0.5(Q=0.135, \varepsilon=0.0107)$; (b) $D_{x}=0.5, D_{s}=0.3, \gamma=10$, and $\alpha=0.5(Q=0.275, \varepsilon=0.0355)$; and (c) $D_{x}=0.1, D_{s}=0.1, \gamma=5$, and $\alpha=0.1(Q=0.011$, $\varepsilon=0.00255)$. The inset in (a) is plotted on a log scale $(x \geq 0)$ and the others on linear scales.

where $I_{n}(z)$ is the modified Bessel function of the first kind and $C$ is to be numerically evaluated by the normalization condition.

Figure 3 shows the stationary distributions calculated by the three methods for the quartic bistable potential case. The meaning of each symbol is the same as in Fig. 2, We also see that the densities of the HFPE at stable sites $(x=-1$ and 1$)$ are higher than those of the FPE under the existence of noise-intensity fluctuations. The inset in Fig. 3(a) (a log-scale plot) shows that the distribution has fatter tails under noise-intensity fluctuations. As in the case of the parabolic potential, the stationary distributions under additive SIN are not Boltzmann-Gibbs distributions. According to the RMS values, we also see a good agreement between the HFPE and MC results. From the log-scale plot, the HFPE result shows better agreement than the FPE also in tail areas for sufficiently large $\gamma$ and sufficiently small $\varepsilon$. 

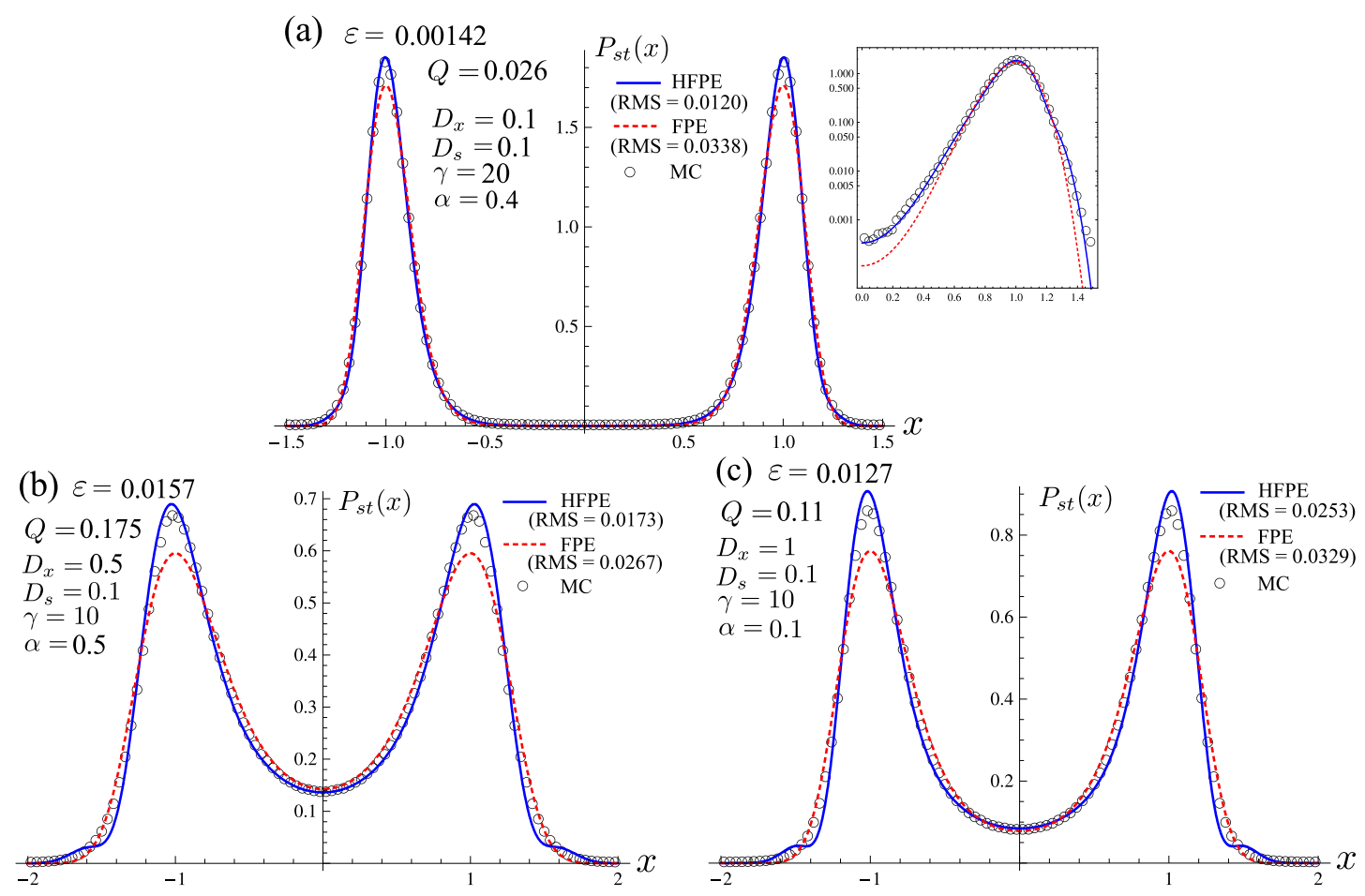

FIG. 3: (Color online) Stationary distributions of the quartic bistable potential with additive noise calculated by HFPE (solid lines), FPE (dashed lines), and MC (circles). Parameter values are (a) $D_{x}=0.1, D_{s}=0.1, \gamma=20$, and $\alpha=0.4(Q=0.026, \varepsilon=0.00142)$; (b) $D_{x}=0.5, D_{s}=0.1$, $\gamma=10$, and $\alpha=0.5(Q=0.175, \varepsilon=0.0157) ;$ and $(\mathrm{c}) D_{x}=1, D_{s}=0.1, \gamma=10$, and $\alpha=0.1$ $(Q=0.11, \varepsilon=0.0127)$. The inset in (a) is plotted on a log scale $(x \geq 0)$ and the others on linear scales.

\subsection{Quartic Bistable Potential with Multiplicative Noise}

We consider the quartic bistable potential as in the previous section $\left[U(x)=x^{4} / 4-\right.$ $\left.x^{2} / 2\right]$, but the system in this section is driven by linear multiplicative noise $[g(x)=x]$. Multiplicative noise plays an important role in many phenomena. $\Pi_{0}(x)$ and $\Pi_{1}(x)$ are given by

$$
\begin{gathered}
\Pi_{0}(x)=\frac{1}{Z}|x|^{\frac{1-Q}{Q}} \exp \left(-\frac{x^{2}}{2 Q}\right), \\
\Pi_{1}(x)=C|x|^{\frac{1-Q}{Q}} \exp \left(-\frac{x^{2}}{2 Q}\right)+\frac{1}{Z Q^{3}}|x|^{\frac{1-Q}{Q}} \exp \left(-\frac{x^{2}}{2 Q}\right) \\
\times\left\{\frac{x^{6}}{6}-\frac{3}{4}(2 Q+1) x^{4}+\frac{1}{2}\left(4 Q^{2}+6 Q+3\right) x^{2}-\log |x|\right\},
\end{gathered}
$$


where $Z$ and $C$ are

$$
\begin{gathered}
Z=(2 Q)^{\frac{1}{2 Q}} \Gamma\left(\frac{1}{2 Q}\right), \\
C=\frac{1}{12 Q^{3}(2 Q)^{\frac{1}{2 Q}} \Gamma\left(\frac{1}{2 Q}\right)}\left\{6 \log (2 Q)+6 \psi\left(\frac{1}{2 Q}\right)-4 Q(Q+3)-11\right\} .
\end{gathered}
$$

Here, $\psi(x)$ is the digamma function $\left[\psi(x)=\partial_{x} \log \Gamma(x)\right]$. We see that $\Pi_{0}(x)$ is composed of two Gamma distributions for $x>0$ and $x<0$. Depending on the effective noise intensity $Q, \Pi_{0}(x)$ is unimodal $(Q \geq 1)$ or bimodal $(0<Q<1)$.

Figure 4 represents the probability densities of three typical cases. Figure 4(a) shows the bimodal stationary distribution $(Q<1)$. In this case, the existence of noise-intensity fluctuations makes densities around $x=1$ and $x=-1$ higher. This is also observed in the additive noise case. Figure 4(b) shows qualitatively different shapes between stationary distributions of FPE and HFPE. Because $Q=0.96<1$ in Fig. 4(b), the FPE stationary distribution (dashed line) exhibits bimodality. On the other hand, the stationary distribution of HFPE is unimodal (this result agrees with the MC simulation). This indicates that the transition from bimodal to unimodal is induced by the existence of noise-intensity fluctuations, and the FPE stationary distribution does not correctly reflect this property. Figure 4(c) also shows the qualitatively different results between FPE and HFPE stationary distributions. It is interesting to see that the HFPE stationary distribution is trimodal, which is not observed without the existence of noise-intensity fluctuations (the MC simulation also exhibits trimodality). This trimodal distribution is a result of superposition of unimodal and bimodal distributions. This result shows that the HFPE formulation is essential to understand systems driven by multiplicative noise. In all figures, the RMS values show that the HFPE distributions provide more reliable results than FPE distributions. From the inset in Fig. 4(a) (a log-scale plot), we see that the GPFE distribution agrees with the MC distribution for tail areas for sufficiently large $\gamma$ and sufficiently small $\varepsilon$.

\subsection{Gene Expression Model}

Langevin equations are often used for describing stochastic chemical reactions. In order to show that our approximation scheme can be applied to systems including non-trivial drift terms, we apply it to a stochastic gene expression model [34]. A simple genetic expression 


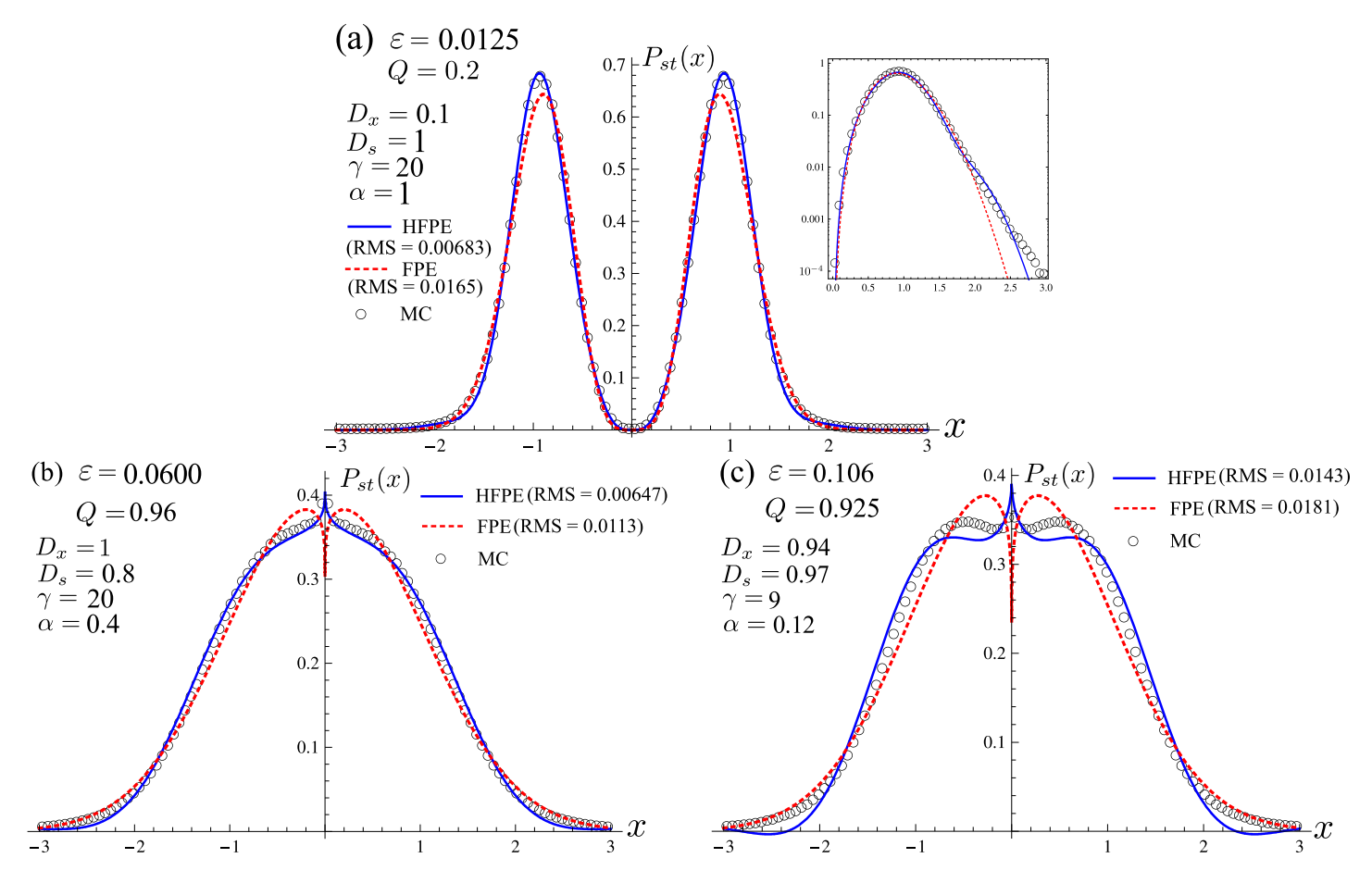

FIG. 4: (Color online) Stationary distributions of the quartic bistable potential with linear multiplicative noise $[g(x)=x]$ calculated by HFPE (solid lines), FPE (dashed lines), and MC (circles). Parameter values are (a) $D_{x}=0.1, D_{s}=1, \gamma=20$, and $\alpha=1(Q=0.2, \varepsilon=0.0125)$; (b) $D_{x}=1$, $D_{s}=0.8, \gamma=20$, and $\alpha=0.4(Q=0.96, \varepsilon=0.06)$; and (c) $D_{x}=0.94, D_{s}=0.97, \gamma=9$, and $\alpha=0.12(Q=0.925, \varepsilon=0.106)$. The inset in (a) is plotted on a log scale $(x \geq 0)$ and the others on linear scales.

model with self-regulation is given by

$$
\frac{d x}{d t}=\frac{a x^{2}}{x^{2}+k}-b x+c
$$

where $x$ is the concentration of a transcription factor (protein), and $a, b, c$, and $k$ are model parameters taking positive values (for details, see Ref. [34]). In this model, the potential function is given by

$$
U(x)=a \sqrt{k} \arctan \left(\frac{x}{\sqrt{k}}\right)+\frac{b x^{2}}{2}-(a+c) x .
$$

The units of $a, b$, and $c$ are $\min ^{-1}$, implying that the decay time of Eq. (22) is on the order of minutes. Because gene expression goes through various fluctuations, some of the fluctuations have faster decay times than $x$. Then the model can be cast in the form of 


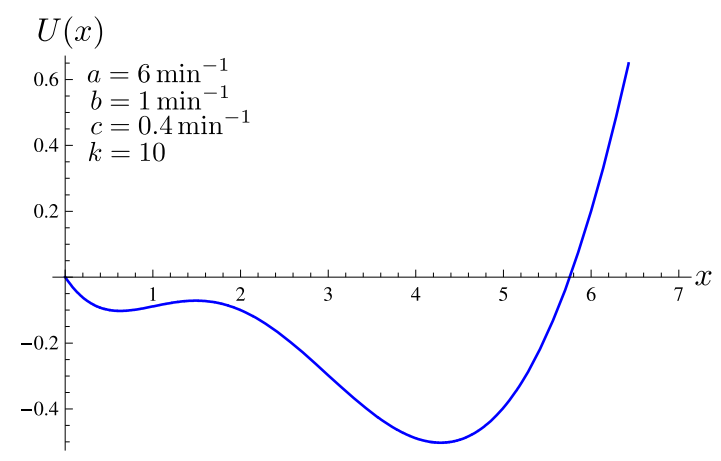

FIG. 5: (Color online) A potential function $U(x)$ for the gene expression model with $a=6 \mathrm{~min}^{-1}$, $b=1 \mathrm{~min}^{-1}, c=0.4 \mathrm{~min}^{-1}$, and $k=10 . U(x)$ exhibits bistability with these parameters.

Langevin equation (3) with

$$
\begin{gathered}
f(x)=\frac{a x^{2}}{x^{2}+k}-b x+c, \\
g(x)=1 .
\end{gathered}
$$

Specifically, we employed $a=6 \mathrm{~min}^{-1}, b=1 \mathrm{~min}^{-1}, c=0.4 \mathrm{~min}^{-1}$, and $k=10$, as in Ref. [22]. With the adopted parameter values, Eq. (23) exhibits bistability (Fig. [5). It is considered that this bistability is responsible for the genetic switch.

Using our approximation method, $\Pi_{0}(x)$ and $\Pi_{1}(x)$ are given as follows:

$$
\begin{aligned}
& \Pi_{0}(x)=\frac{1}{Z} \exp \left\{-\frac{U(x)}{Q}\right\} \\
& \Pi_{1}(x)= C \exp \left(-\frac{U(x)}{Q}\right)-\frac{1}{8 Q^{3} Z} \exp \left(-\frac{U(x)}{Q}\right) \\
& \times\left[-2 b^{3} x^{4}+8 b^{2}(a+c) x^{3}+12 b\left(-a^{2}-2 c a-c^{2}+b Q\right) x^{2}\right. \\
&+ 8\left\{a^{3}+3 c a^{2}+3\left(c^{2}-b(b k+Q)\right) a+c^{3}-3 b c Q\right\} x \\
&+ \frac{3 a k}{x^{2}+k}\left\{3 x a^{2}+4(b k-2 Q+c x) a-8 Q(c-b x)\right\} \\
&-3 a \sqrt{k}\left(5 a^{2}+12 c a+8 c^{2}-8 b^{2} k\right) \arctan \left(\frac{x}{\sqrt{k}}\right) \\
&+\left.24 a b(a+c) k \log \left(x^{2}+k\right)+\frac{2 a k}{\left(x^{2}+k\right)^{2}}\left(-k x a^{2}+6 k Q a+8 Q^{2} x\right)\right],
\end{aligned}
$$

where $Z$ and $C$ are normalizing constants, which are numerically evaluated so that $\int d x \Pi_{0}(x)=1$ and $\int d x P_{s t}^{(1)}(x)=1$. Although the concentration $x$ cannot take negative values, the obtained stationary distribution has a very small magnitude in the $x<0$ areas, which can be ignored in practical calculations. 
(a)

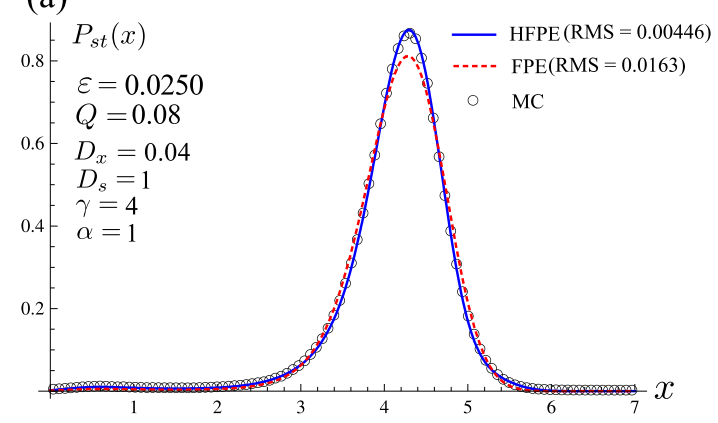

(b)

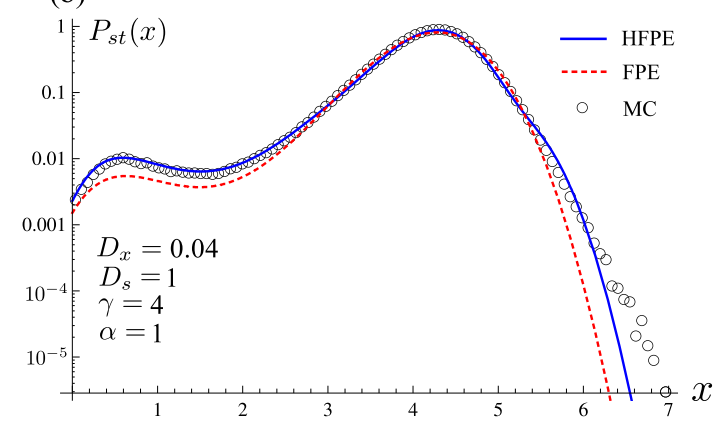

FIG. 6: (Color online) Stationary distributions of the stochastic gene expression model calculated by HFPE (solid lines), FPE (dashed lines), and MC (circles). Parameter values are $D_{x}=0.04$, $D_{s}=1, \gamma=4$, and $\alpha=1(Q=0.08, \varepsilon=0.025)$. (a) is plotted on a linear scale and (b) is on a log scale.

Figure 6 represents probability distributions calculated by the three methods. The distributions in (a) and (b) are plotted on linear and log scales, respectively. We see that the density of the HFPE (solid line) at the stable site $(x \simeq 4.3)$ is higher than the case without noise-intensity fluctuations (dashed line). From the RMS values in Fig. 6(a), the HFPE stationary distribution agrees well with the MC simulations. In the tail areas [Fig. 6(b)], the HFPE result exhibits good agreement with the MC simulations, whereas the FPE result does not. This result indicates that our approximation method can be applied to systems with non-trivial drift terms.

\section{DISCUSSION}

\subsection{Effects of higher-order derivative terms}

In the projected time evolution equations of our model, recursive expansion leads to equations having an infinite number of terms. In this paper, we have truncated at $O\left(\gamma^{-1}\right)$, including derivatives only up to fourth order. Pawula's theorem [35] indicates that to guarantee the positivity of the distribution functions, the truncation must be after the first- or second-order term (this corresponds to FPE). Otherwise, the equations must include an infinite number of terms. However, it has been reported that time evolution equations truncated at $n \geq 3$ are practically meaningful [36 38]. Indeed our approximation including derivatives of higher order than two have shown better results than FPE solutions. In particular, it 
has been shown in Figs. 4(b) and (c) that stationary distributions of FPE and HFPE have qualitatively different shapes, and our results using HFPE are supported by MC simulations.

Ref. [39] gives an intuitive explanation of the effect of higher-order derivatives, which are not included in the conventional FPE, on the positivity of solutions. Following Ref. [39], we explain the parabolic potential case. Using Eq. (13), the FPE of the parabolic potential case is given by

$$
\frac{\partial}{\partial t} P(x ; t)=\left\{\frac{\partial}{\partial x} x+Q \frac{\partial^{2}}{\partial x^{2}}\right\} P(x ; t) .
$$

Let $x_{m}$ be points where $P(x ; t)$ is locally minimal with respect to $x$. According to requirements of the minima and the positivity of $P\left(x_{m} ; t\right), \partial_{t} P\left(x_{m} ; t\right)$ is always positive:

$$
\frac{\partial}{\partial t} P\left(x_{m} ; t\right)=\left.x \frac{\partial}{\partial x} P(x ; t)\right|_{x=x_{m}}+P\left(x_{m} ; t\right)+\left.Q \frac{\partial^{2}}{\partial x^{2}} P(x ; t)\right|_{x=x_{m}}>0 .
$$

Eq. (28) guarantees the positivity of initially positive solutions because at the minima $P\left(x_{m} ; t\right)$ is increasing as a function of $t$. On the other hand, the positivity of $\partial_{t} P\left(x_{m} ; t\right)$ is not generally satisfied in HFPE cases, since the fourth-order derivative term $\left.(R / \gamma) \partial_{x}^{4} P(x ; t)\right|_{x=x_{m}}$ can take any values. However, the effect of the fourth-order term on the sign of $\partial_{t} P\left(x_{m} ; t\right)$ is negligible under sufficiently smooth initial distributions and sufficiently large $\gamma$, since the fourth-order term is on the order of $\gamma^{-1}$. From the above explanation, it is generally expected that solutions of HFPE can satisfy positivity under sufficiently large $\gamma$.

In order to analyze effects of higher-order derivatives on moments for the additive noise case, we consider the following moment expansion using Eq. (12):

$$
\frac{\partial}{\partial t}\left\langle x^{n}\right\rangle=n\left\langle f(x) x^{n-1}\right\rangle+Q n(n-1)\left\langle x^{n-2}\right\rangle+\frac{R}{\gamma} n(n-1)(n-2)(n-3)\left\langle x^{n-4}\right\rangle,
$$

where $\langle\cdots\rangle$ represents the expectation with respect to $P(x ; t)\left[\langle A(x)\rangle=\int d x A(x) P(x ; t)\right]$. We consider the stationary case of the parabolic potential case $[f(x)=-x]$. Equation (29) in this case is described by

$$
\langle x\rangle=0, \quad\left\langle x^{2}\right\rangle=Q, \quad\left\langle x^{3}\right\rangle=0, \quad\left\langle x^{4}\right\rangle=3 Q^{2}+\frac{6 R}{\gamma} .
$$

Kurtosis is given by

$$
\kappa=\frac{\left\langle x^{4}\right\rangle}{\left\langle x^{2}\right\rangle^{2}}-3=\frac{6 D_{s}\left(4 \alpha^{2}+D_{s}\right)}{\gamma\left(D_{s}+\alpha^{2}\right)^{2}} .
$$

$\gamma \rightarrow \infty$ yields the Gaussian stationary distribution with $\kappa=0$. Equation (30) shows that smaller $\gamma$ yields a stationary distribution with larger kurtosis. This means that the distributions have fatter tails under noise-intensity fluctuations. This result agrees with those of 
Sec. [5.1, In Ref. [13], fat-tailed stationary distributions are derived not from time evolution equations but from a calculation of the expectation with respect to a noise-intensity distribution. Our results show that derivatives of orders higher than two are required to yield fat-tailed stationary distributions in the parabolic potential. Furthermore, the importance of the fourth-order derivative was pointed out in Ref. [20], which considered superstatistical Brownian particles using mesoscopic nonequilibrium thermodynamics [40]. Ref. [20] obtained a time evolution equation in the Fourier space. Inclusion of a quartic term in the terms of a Fourier conjugate variable indicates the existence of a fourth-order derivative in real space. In specific cases, stationary distributions of their model are very close to a q-Gaussian distribution, which are strongly connected to Beck's superstatistics.

The stationary distributions of the FPE (Sec. 5.1) belong to the exponential family, which are strongly connected to the Boltzmann-Gibbs-Shannon (BGS) entropy. On the other hand, the stationary distributions of the HFPE with fatter tails are not exponential. Nonexponential distributions in statistical mechanics have been extensively discussed in Tsallis statistics [15, 16]. It was shown that long-range interactions or long-time memory effects are responsible for such distributions. Since the noise intensity of our model is governed by the Ornstein-Uhlenbeck process, correlation time of the noise intensity serves as the memory effect, which is not the case for the white noise.

\subsection{Relation to colored noise}

As argued in Sec. 1, colored noise is a generalization of white noise and also incorporates mesoscopic time-scale inhomogeneity. However, many approximation schemes for colored noise, including adiabatic elimination, can take into account a time-correlation effect [i.e., $O(\tau)$ ] within the conventional FPE. The necessity of derivatives of higher order than two in our systems indicates that systems driven by SIN are essentially different than those governed by colored noise.

For colored noise driven systems, one popular approach to obtain approximate FPE is the unified colored-noise approximation (UCNA), which is based on the adiabatic elimination approach [26]. In Refs. [41, 42], UCNA was confirmed as a reliable Markovian approximation by the path-integral. In Refs. [41, 42], they derived FPE using a Markovian Lagrangian function in the path-integral representation, where non-Markovian terms, such as $\ddot{x}$ and 

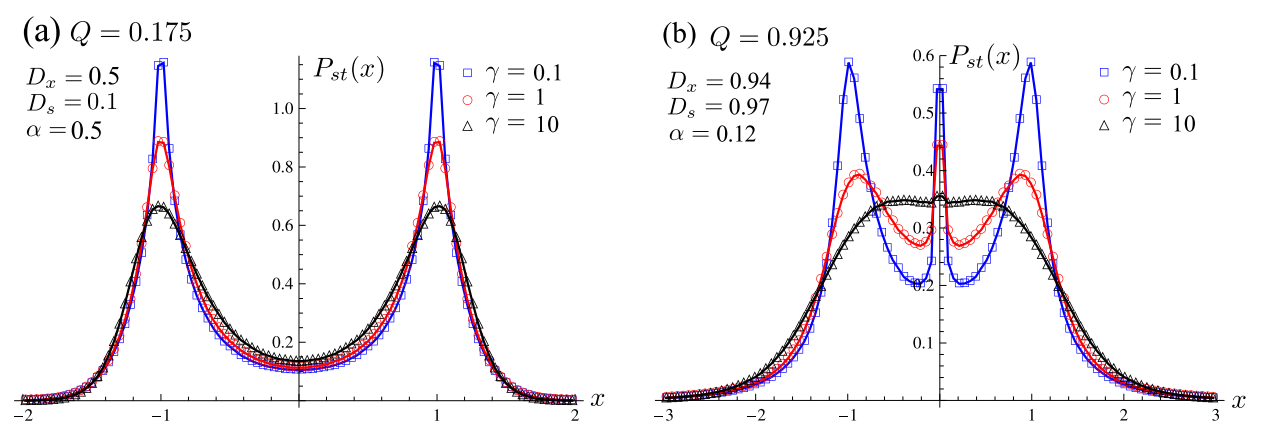

FIG. 7: (Color online) Stationary distributions of the quartic bistable potential with (a) additive and (b) multiplicative noise for $\gamma=0.1$ (squares), 1 (circles) and 10 (triangles). Parameter values are (a) $D_{x}=0.5, D_{s}=0.1$, and $\alpha=0.5(Q=0.175)$ and (b) $D_{x}=0.94, D_{s}=0.97$, and $\alpha=0.12$ $(Q=0.925)$. These data are from MC simulations with $N=5 \times 10^{7}$ samples. Solid lines are included as a guide to the eye only.

$\dot{x}^{n}(n \geq 2)$, were ignored. The path-integral was applied to non-Gaussian colored noise in Ref. [43], and an effective Markovian FPE was obtained in this case. Since our obtained equation includes derivatives of higher order than two, a naive application of the pathintegral techniques used in the colored noise case may not be possible. However, it is important to understand our approximation scheme from the viewpoint of the path-integral, because the path-integral has an intuitive interpretation in stochastic processes. We leave these for future studies.

\subsection{Cases of the other $\gamma$ range}

In preceding sections, we described the approximation scheme for the $\gamma \gg 1$ case. It is important to consider the other $\gamma$ range. In this section, we describe MC simulations carried out for $\gamma=0.1,1$, and 10 for two settings: the quartic bistable potential with additive and with multiplicative noise. Parameters $\left(D_{x}, D_{s}\right.$, and $\left.\alpha\right)$ of the additive and multiplicative noise cases are identical to those shown in Figs. 3(b) and 4(c), respectively. These results are shown in Fig. 7. Fig. 7(a) shows that higher peaks emerge at $x= \pm 1$ for smaller $\gamma$. For Fig. 7(b), densities at $x=-1,0$, and 1 are higher for smaller $\gamma$. Similar trends have also been observed in Secs. 5.1 and 5.2. Results of Sec. 5.2 and this section show that trimodality is induced when the noise-intensity fluctuations are meso/macroscopic and the fluctuation width is large. The stationary distributions of smaller $\gamma$ can be explained 
TABLE I: RMS distance between HFPE (or FPE) and MC distributions for parabolic and quartic bistable potentials with additive noise, quartic potential with multiplicative noise, and a gene expression model.

\begin{tabular}{|c|c|c|c|c|c|c|}
\hline \multirow{2}{*}{} & \multicolumn{2}{|c|}{$\begin{array}{c}\text { parabolic potential } \\
\text { with additive noise }\end{array}$} & \multicolumn{2}{c|}{$\begin{array}{c}\text { quartic bistable potential } \\
\text { with additive noise }\end{array}$} \\
\cline { 2 - 7 } & Fig. 2(a) & Fig. 2(b) & Fig. 2(c) & Fig. 3(a) & Fig. 3(b) & Fig. 3(c) \\
\hline \hline HPFE & 0.00418 & 0.00788 & 0.0934 & 0.0120 & 0.0173 & 0.0253 \\
\hline FPE & 0.0184 & 0.0229 & 0.152 & 0.0338 & 0.0267 & 0.0329 \\
\hline
\end{tabular}

\begin{tabular}{|c|c|c|c|c|}
\hline & \multicolumn{2}{|c|}{$\begin{array}{c}\text { quartic bistable potential } \\
\text { with multiplicative noise }\end{array}$} & gene expression \\
& Fig. 4(a) & Fig. 4(b) & Fig. 4(c) & Fig. 6 \\
\hline \hline HPFE & 0.00683 & 0.00647 & 0.0143 & 0.00446 \\
\hline FPE & 0.0165 & 0.0113 & 0.0181 & 0.0163 \\
\hline
\end{tabular}

by the superposition of different distributions. For $\gamma \rightarrow 0$, the case reduces to that of superstatistics, where the stationary distribution is given in a Bayesian fashion:

$$
P_{s t}(x) \sim \int d s P_{s t}(x \mid s) P(s) .
$$

Here, $P_{s t}(x \mid s)$ is the stationary distribution taking $s$ as a parameter, and $P(s)$ is the distribution of $s$.

Ref. [18] considered a different superstatistical model of multiplicative noise processes:

$$
\frac{d x}{d t}=f(x)+\left[x^{2}\right]^{\alpha} \xi(t)
$$

which assumes that multiplicative noise exponent $\alpha$ fluctuates $[\xi(t)$ is white noise]. Stationary distributions of their model also exhibit the transitions of stationary distributions.

\section{CONCLUDING REMARKS}

In this paper, we have derived the time evolution equation of systems driven by SIN using adiabatic elimination under the assumption $\gamma \gg 1$. The obtained HFPE is of $O\left(\gamma^{-1}\right)$ and contains derivatives up to fourth order. We have calculated the stationary distributions 
of the obtained equation by using the perturbation expansion and applied them to three different cases. Table \summarizes the RMS distance between the stationary distributions calculated by the HFPE (or FPE) and MC which have been shown in Figs. 2, 3, 4, and 6, It clearly shows that the results of the HFPE are better than those of FPE. We have pointed out that SIN makes densities at stable sites higher. Furthermore, SIN induces the transition from unimodal to bimodal in the quartic bistable potential driven by multiplicative noise. For specific parameters, the stationary distribution exhibits three peaks, which are not observed in conventional white noise cases. Because the stationary distributions obtained by Eqs. (17) and (18) are given by the first-order differential equations, their analytical expressions can be calculated for many general configurations. The applicability has been shown with the stochastic gene expression model, which has a non-trivial drift term. This indicates that our method can be applied to many real-world phenomena. Furthermore, higher-order derivatives in HFPE have been given much attention in recent years [39, 44]. Analysis of higher-order terms in our systems are also important, and so we intend to pursue this in future studies.

\section{Acknowledgments}

This work was supported by a Grant-in-Aid for Scientific Research on Priority Areas "Systems Genomics" from the Ministry of Education, Culture, Sports, Science and Technology, Japan.

\section{Appendix A: Derivation of the Higher-Order Fokker-Planck Equation}

In this appendix, we explain the procedures of adiabatic elimination with eigenfunction expansion used in our model, following Refs. [23, 33].

We first expand $P(x, s ; t)$ into the complete set $\psi_{n}(s)$ of the operator $\mathrm{L}_{s}(s)$ [Eq. (9)]:

$$
P(x, s ; t)=\sum_{n=0}^{\infty} P_{n}(x ; t) \psi_{n}(s),
$$

where $\psi_{n}(s)$ are eigenfunctions of the following equation:

$$
\mathrm{L}_{s}(s) \psi_{n}(s)=-\lambda_{n} \psi_{n}(s)
$$


Eigenvalues $\lambda_{n}$ and eigenfunctions $\psi_{n}(s)$ are given by

$$
\begin{gathered}
\lambda_{n}=n, \\
\psi_{n}(s)=\sqrt{\frac{1}{2 \pi D_{s}}} \frac{1}{2^{n} n !} H_{n}(\eta) \exp \left(-\eta^{2}\right),
\end{gathered}
$$

where $\eta=\sqrt{1 /\left(2 D_{s}\right)}(s-\alpha)$, and $H_{n}(\eta)$ is the $n$th Hermite polynomial. We introduce the adjoint function $\psi_{n}^{\dagger}(s)$ of $\psi_{n}(s)$ :

$$
\psi_{n}^{\dagger}(s)=H_{n}(\eta), \quad \psi_{0}^{\dagger}(s)=1
$$

The orthonormality and complete relations are

$$
\left\langle\psi_{n}^{\dagger}(s) \psi_{m}(s)\right\rangle=\delta_{n m}
$$

where $\langle\cdots\rangle$ denotes an integration with respect to $s\left[\langle A(s)\rangle=\int d s A(s)\right]$. Using Eqs. (A44) and ( $(\underline{\mathrm{A} 5}), P(x, t)(=\langle P(x, s, t)\rangle)$ is given by

$$
P(x ; t)=\sum_{n=0}^{\infty} P_{n}(x ; t)\left\langle\psi_{0}^{\dagger}(s) \psi_{n}(s)\right\rangle=P_{0}(x ; t) .
$$

By multiplying both sides of Eq. (6) by $\psi_{m}^{\dagger}(s)$ and integrating out $s$, we obtain $\left[P_{m}=\right.$ $\left.P_{m}(x ; t)\right]$

$$
\frac{\partial}{\partial t} P_{m}=-\frac{\partial}{\partial x} f P_{m}+D_{x} \sum_{n=0}^{\infty}\left\langle\psi_{m}^{\dagger} s^{2} \psi_{n}\right\rangle \triangle_{g} P_{n}-\gamma \lambda_{m} P_{m},
$$

where $\triangle_{g}$ is an operator defined by Eq. (11).

Using relations of the Hermite polynomial, specifically, $H_{n+1}(z)=2 z H_{n}(z)-2 n H_{n-1}(z)$ and $H_{0}(z)=1$, the following relation holds:

$$
\begin{aligned}
\left\langle\psi_{m}^{\dagger}(s) s^{2} \psi_{n}(s)\right\rangle= & 2 D_{s} m(m-1) \delta_{m-2, n}+2 \sqrt{2 D_{s}} \alpha m \delta_{m-1, n}+\frac{1}{2} D_{s} \delta_{m+2, n} \\
& +\sqrt{2 D_{s}} \alpha \delta_{m+1, n}+\left\{2 D_{s}\left(m+\frac{1}{2}\right)+\alpha^{2}\right\} \delta_{m, n} .
\end{aligned}
$$

According to Eqs. (A7) and (A8), time evolution of $P_{0}$ is governed by

$$
\frac{\partial}{\partial t} P_{0}=-\frac{\partial}{\partial x} f P_{0}+D_{x}\left(D_{s}+\alpha^{2}\right) \triangle_{g} P_{0}+\sqrt{2 D_{s}} \alpha D_{x} \triangle_{g} P_{1}+\frac{1}{2} D_{s} D_{x} \triangle_{g} P_{2} .
$$

Equation (A9) includes $P_{1}$ and $P_{2}$, although we want to obtain a closed equation of $P_{0}$. We calculate $P_{m}(m \geq 1)$ by Eq. (A7):

$$
P_{m}=\frac{1}{\lambda_{m} \gamma}\left\{-\frac{\partial}{\partial x} f P_{m}+D_{x} \sum_{n=0}^{\infty}\left\langle\psi_{m}^{\dagger} s^{2} \psi_{n}\right\rangle \triangle_{g} P_{n}\right\} \quad(\text { for } m \geq 1)
$$


In Eq. (A10), we ignore a time derivative term. By substituting Eq. (A10) into Eq. (A9), up to $O\left(\gamma^{-1}\right)$, we obtain Eq. (10).

[1] C. R. Doering, J. C. Gadoua, Phys. Rev. Lett. 69 (1992) 2318.

[2] R. N. Mantegna, B. Spagnolo, Phys. Rev. Lett. 76 (1996) 563.

[3] A. A. Dubkov, N. V. Agudov, Phys. Rev. E 69 (2004) 061103.

[4] B. Spagnolo, A. A. Dubkov, N. V. Agudov, Eur. Phys. J. B 40 (2004) 273.

[5] B. Spagnolo, N. V. Agudov, A. A. Dubkov, Acta Phys. Pol. B 35 (2004) 1419.

[6] A. Fiasconaro, B. Spagnolo, Phys. Rev. E 80 (2009) 041110.

[7] S. L. Heston, Rev. Financ. Stud. 6 (1993) 327.

[8] A. A. Drăgulescu, V. M. Yakovenko, Quant. Finance 2 (2002) 443.

[9] G. Bonanno, D. Valenti, B. Spagnolo, Phys. Rev. E 75 (2007) 016106.

[10] G. Wilk, Z. Włodarczyk, Phys. Rev. Lett. 84 (2000) 2770.

[11] C. Beck, Phys. Rev. Lett. 87 (2001) 180601.

[12] C. Beck, E. G. D. Cohen, Physica A 322 (2003) 267.

[13] C. Beck, Prog. Theor. Phys. Supp. 162 (2006) 29.

[14] C. Beck, Braz. J. Phys. 39 (2009) 357.

[15] C. Tsallis, J. Stat. Phys. 52 (1988) 479.

[16] C. Tsallis, Introduction to Nonextensive Statistical Mechanics: Approaching a Complex World, Springer, 2009.

[17] P. Jizba, H. Kleinert, Phys. Rev. E 78 (2008) 031122.

[18] S. M. D. Queirós, Braz. J. Phys. 38 (2008) 203.

[19] Y. Hasegawa, M. Arita, Physica A 389 (2010) 4450.

[20] R. F. Rodríguez, I. Santamaría-Holek, Physica A 385 (2007) 456.

[21] S. M. D. Queirós, C. Tsallis, Eur. Phys. J. B 48 (2005) 139.

[22] Q. Liu, Y. Jia, Phys. Rev. E 70 (2004) 041907.

[23] K. Kaneko, Prog. Theor. Phys. 66 (1976) 129.

[24] W. Horsthemke, R. Lefever, Noise-Induced Transitions, Springer, 1984.

[25] C. W. Gardiner, Phys. Rev. A 29 (1984) 2814.

[26] P. Jung, P. Hänggi, Phys. Rev. A 35 (1987) 4464. 
[27] R. Remer, R. Mahnke, Physica A 344 (2004) 236.

[28] T. S. Biró, R. Rosenfeld, Physica A 387 (2008) 1603.

[29] R. Kubo, K. Matsuo, K. Kitahara, J. Stat. Phys. 9 (1973) 51.

[30] W. Horsthemke, R. Lefever, Z. Physik B 40 (1980) 241.

[31] C. Knessl, M. Mangel, Z. Schuss, C. Tier, J. Chem. Phys. 81 (1984) 1285.

[32] T. D. Frank, Phys. Rev. E 71 (2005) 031106.

[33] H. Risken, The Fokker-Planck Equation: Methods of Solution and Applications, 2nd Edition, Springer, 1989.

[34] P. Smolen, D. A. Baxter, J. H. Byrne, Am J Physiol 274 (1998) C531.

[35] R. F. Pawula, Phys. Rev. 162 (1967) 186.

[36] U. M. Titulaer, Physica 91A (1978) 321.

[37] H. Risken, H. D. Vollmer, Z. Phys. B 35 (1979) 313.

[38] H. Risken, H. D. Vollmer, Z. Phys. B 66 (1987) 257.

[39] A. V. Plyukhin, Physica A 351 (2005) 198.

[40] P. Mazur, Physica A 274 (1999) 491.

[41] P. Colet, H. S. Wio, M. S. Miguel, Phys. Rev. A 39 (1989) 6094.

[42] H. S. Wio, P. Colet, M. S. Miguel, L. Pesquera, M. A. Rodríguez, Phys. Rev. A 40 (1989) 7312.

[43] M. A. Fuentes, H. S. Wio, R. Toral, Physica A 303 (2002) 91.

[44] A. V. Plyukhin, Phys. Rev. E 77 (2008) 061136. 